\title{
In Pursuit of the IDEAL Fit
}

\author{
Joanna GOULD-THORPE* \\ Me-Ality, Unique Solutions Ltd., Darthmouth (NS), Canada
}

\section{In pursuit of the IDEAL fit}

Me-Ality is the size matching service that allows you to find your best fitting clothing items from a multitude of brands without ever walking into a store or stepping into a fitting room. We take a quick ten second scan of your body and provide you with a shopping guide of the sizes, styles and brands that best fit your unique body type. Never try on several different sizes or return an online order again.

Self-image and self-worth are tied more closely to having our clothes fit well than we ever understood before. I have observed how women personalize the "number" on clothing tags; a size number up or down affects how they feel about themselves. Yet, those numbers are increasingly irrelevant. What is a size 8 ? We jokingly refer to the 8 million size 8's out there because you can take many different women of many different shapes and proportions and all of them can fall into some brands' size 8 but never consistently into every brand's size 8 . The reality is that without consistency in sizing across brands, the consumer will continue to be confused. Scanning and fitting technologies have a very real opportunity to solve this serious challenge for both consumers and retailers alike. Technologies like Me-Ality's Size Matching Service built on a foundation of scanned measurements are solving this challenge for consumers today.

Dressing room drama is a tongue in cheek way of describing the potentially soul crushing experience that was the dressing room for many women in the past. How are scanning and fitting technologies taking away the pain of the dressing room? No woman wants to try on clothes that don't fit. It doesn't matter if you find one that fits in the end, the exercise of trying on what feels like an endless selection of garments and the frustration of looking at the 8 discarded items on the bench while you hold one in your hands is demoralizing enough. Imagine as a retailer that the first item a consumer tries on in the dressing room fits; now imagine how much more willing that same consumer is to try on other garments to go with it. It's not just her time that's been maximized, but her well-being. She's more confident, she's enthusiastic, she's ready to spend money and the use of scanning and fit technologies have created the environment for her to do that in. Extend that to the online consumer and you compound both the current challenge and the potential benefit for retailers. Right now approximately $75 \%$ of clothing returned to online retailers is due to lack of fit. Consumers intentionally order more than one size knowing that they will return at least one if not all. The customer who shops online and in-store is worth $200 \%$ more than a customer who shops at just one or the other. The impact of scanning and providing fit recommendations for these consumers is huge.

But how good are fitting technologies? Is scanning accuracy good enough to provide consumers with accurate sizing and fitting recommendations?

Accuracy in scanning technologies is a hot topic. Accurate to two $\mathrm{mm}$; accurate to 1/16th of an inch; accurate to $+/-.5 \mathrm{~cm}$, all of these have value to those of us using scanning technology for applications, inventions or manufacturing processes. Fitting technologies rely on scanning accuracy to create sizing recommendations or suggestions, but is that all that matters? In apparel sizing accuracy of the scan is relevant, no question. But accuracy or the perception of accuracy is perceived by the consumer in its impact of how the products recommended by scanning make them feel. This depends not only on the dynamics of the body but the area of the body being fit, and the individual and their preference of fit.

Consider the dynamics of different areas of the body. Some parts are hard and angular; shoulders and feet, others softer, more malleable, "squishy", if you will, such as waists and thighs. 
For example, there are more bones in your foot than most other areas of the body. This makes it relatively easy to get a clean scan with hard angular edges that can be measured very accurately. Are these measurements the most important thing in the fit of the shoe needed by that foot? I know of custom shoe manufacturers that could make the most precise fitting shoe you could imagine. It would fit so well that not even a slip of paper would get between you and the rich Italian leather, but one step and you would be slipping out of them at the heel because it's not just the measurements that matter, it's the combination of measurements, break points, last construction and the end use that make a shoe an ideal fit.

Another factor to consider is that bodies with the exact same measurement may fit into different sizes because of the "squish factor" in their bodies. An athletic, muscled form with a waist measurement of 28 inches has much less tolerance for accuracy of fit than a soft curvy form with the same measurement. Some bodies can naturally tolerate more squish in their apparel and still find comfort and perceived accuracy in the fit. In addition to this tolerance between body types there is the specific measurement location on any given body that has to be considered as well. If you make pants too tight in an area where fluid motion is happening they may fit correctly, but it won't allow the movement needed to allow the individual to feel like it fits. These dynamics influence the overall perception of accuracy in scan measurements but that's just it. It's the perception of accuracy.

Let's layer in something else; ease. I don't want to assume that everyone here understands ease in apparel and why and how it's applied so if you'll indulge me: Ease is the amount of extra room drafted into a pattern over and above body measurements. There are two types of ease: 1) Basic ease is the amount added to a pattern for comfort, movement, sitting, standing, eating, breathing etc. 2) Design ease: this is the amount of extra inches designers add to a pattern to create the "look" they want. Different people have different preferences for the amount of ease they find comfortable in a garment and therefore their perception of fit includes a factor that is relevant only to them and their own personal preference.

Between basic ease and design ease the garment could have an almost infinite difference between the actual body measurement of a person and the garment measurement in a given area. We could debate how much difference is enough, how much is too much and engage every designer in every brand across the globe without consensus, so the difference of a half centimeter in scanning accuracy isn't going to be the big differentiating factor here. At the end of the day scanning technologies take measurements accurate enough to base garment manufacturing and fitting technologies on, but fitting technologies need to be aware of and accommodate for all of the other factors noted above.

Let's come back to self-image.

It's not just fit but style; and the perceived view of what you see affecting your self-esteem. Imagine having a technology telling you something fits and looks good on you; it's like taking a good friend shopping. Only this friend has to tell the truth and you can trust them. Scanning allows us to not only factor in measurements to create fitting recommendations, but because scanning creates a full 3D representation of the body, the amount of visual fit data available to use in style and flattery technologies is abundant. With the right expertise being applied to that data, new technologies can not only guide a consumer to the garment with the best fit, but help them choose the things that will truly flatter them.

It's in all of our best interests to see that scanning, fitting and style technologies become more readily available to the consumer and easier to use. In addition to the obvious benefits to the consumers and the direct benefits to the retailers in sales and merchandising the future benefits are staggering. As they become convenient because of the feedback between the brands, the customers, etc. you can begin to imagine a world where our scanning and fitting technologies can drive a model that provides for better fitting clothes through an iterative process that engages every level of the apparel lifecycle.

It's the evolution of clothing. It's the evolution of retail. It's the evolution of our scanning, size matching and style technologies being used in a meaningful way. 